\title{
Improving diffraction limits by engineering crystallization chaperones - a case study of HIV1 envelope trimer and its application to drug design.
}

\begin{abstract}
Yen-Ting Lai and Peter D. Kwong
Vaccine Research Center, National Institute of Allergy and Infectious Diseases, National Institutes of Health, Bethesda, MD 20892, USA

The envelope (Env) protein of HIV-1 recognizes target cells and mediates cell fusion for viral entry and thus is an important target for drug development. Structures of HIV-1 Env have been determined by protein crystallography and cryo- electron microscopy.

However, the resolutions of published Env structures are in general lower than $3.5 \AA$, which hinders its utility for structure-based drug design. Here, we use lattice engineering to improve the resolution of a previously published crystal structure of HIV-1 Env BG505.SOSIP.664 [1] by engineering improved versions of two crystallization chaperones, $35 \mathrm{O} 22$ and PGT122 Fabs (antigen-binding fragments of antibodies), both present in the previously published lattice. Specifically, we converted $35 \mathrm{O} 22$ Fab to single-chain variable region ( $\mathrm{scFv}$ ) based on the observation that the constant region of the Fab is very flexible and does not make meaningful crystal contacts in the lattice. The $35 \mathrm{O} 22 \mathrm{scFv}$, in combination with 3H/109L Fab (a precursor of PGT122 family of antibodies), led to a crystal lattice with almost identical unit cell parameters and resolution as in the published structure. Further improvement of the resolution of BG505.SOSIP.664 was achieved by introduction of stabilizing mutations on both 35022 scFv and $3 \mathrm{H} / 109 \mathrm{~L} F \mathrm{Fab}$, resulting in diffractions of $\sim 2.4 \AA$ along the best diffracting direction and overall resolution of $\sim 3.0 \AA$. This improved crystal lattice was used for drug soaking from a few different classes of inhibitors targeting Env, including BMS-626529 of which a prodrug is currently in a phase III trial. Significant electron density improvement was achieved compared to soaking experiments conducted in the original lattice, leading to unambiguous modeling of protein side chains interacting with the functional groups of drugs.
\end{abstract}

\section{Reference:}

[1] Pancera, M., et al., Structure and immune recognition of trimeric pre-fusion HIV-1 Env. Nature, 2014. 514(7523): p. 455-61. 